PROCEEDINGS OF THE

AMERICAN MATHEMATICAL SOCIETY

Volume 130, Number 7, Pages 1955-1959

S 0002-9939(02)06503-6

Article electronically published on February 8, 2002

\title{
ON THE REGULARITY OF SOLUTIONS TO FULLY NONLINEAR ELLIPTIC EQUATIONS VIA THE LIOUVILLE PROPERTY
}

\author{
QINGBO HUANG
}

(Communicated by David S. Tartakoff)

\begin{abstract}
We show that any $C^{1,1}$ solution to the uniformly elliptic equation $F\left(D^{2} u\right)=0$ must belong to $C^{2, \alpha}$, if the equation has the Liouville property.
\end{abstract}

\section{$\S 1$. INTRODUCTION}

In this paper, we consider the interior regularity of solutions to the following fully nonlinear elliptic equation:

$$
F\left(D^{2} u\right)=0
$$

We assume that $F$ is uniformly elliptic, i.e., there exist constants $0<\lambda \leq \Lambda$ such that

$$
\lambda\|N\| \leq F(M+N)-F(M) \leq \Lambda\|N\|, \quad \text { for } M, N \in \mathcal{S}, N \geq 0,
$$

where $\mathcal{S}$ denotes the space of real $n \times n$ symmetric matrices and $\|N\|$ denotes the norm of $N$.

For simplicity, we also assume that $F(0)=0$.

There have been a number of works concerning equation (1). For instance, see $\mathrm{CC}, \mathrm{GT},[\mathrm{K}$ and the references cited there. When $F$ is a concave or convex functional, it is well known that the Evans-Krylov estimate

$$
\left[D^{2} u\right]_{C^{\alpha}\left(B_{1 / 2}\right)} \leq C\|u\|_{C^{1,1}\left(B_{1}\right)}
$$

holds, and $C^{1,1}$ viscosity solutions of (1) are $C^{2, \alpha}$ for some $\alpha>0$.

On the contrary, in the case when $F$ is not concave nor convex, $C^{1,1}$ viscosity solutions of (1) may not be in the $C^{2}$ class. This has recently been shown by Nadirashvili in $\mathrm{N}$ in which he found a $C^{1,1}$ viscosity solution $u$ to the equation $F\left(D^{2} u\right)=0$ where $F$ is smooth, uniformly elliptic and $u$ is not $C^{2}$. Therefore, it would be interesting to know under what condition a $C^{1,1}$ solution of (1) is actually in the $C^{2}$ class.

It is our purpose in this paper to show that any $C^{1,1}$ viscosity solution of (1) must be $C^{2, \alpha}$ if the elliptic operator $F$ has the Liouville property.

A continuous function $u(x)$ is said to be a viscosity subsolution (resp., supersolution) of (1) in a domain $\Omega$ if for $x_{0} \in \Omega$ and $\phi(x) \in C^{2}, u-\phi$ attains the local

Received by the editors September 20, 1999.

2000 Mathematics Subject Classification. Primary 35J60; Secondary 35B65.

Key words and phrases. Fully nonlinear elliptic equation, regularity, Liouville property, VMO.

(C)2002 American Mathematical Society 
maximum (resp., minimum) at $x_{0}$, then $F\left(D^{2} \phi\left(x_{0}\right)\right) \geq 0($ resp., $\leq 0)$. If $u$ is both a subsolution and a supersolution, then we say $u$ is a viscosity solution. We mention that if $u \in C^{1,1}$, then $u$ is a viscosity solution of (1) if and only if $u$ is a strong solution to $(1)$.

Equation (1) or $F$ is said to satisfy the Liouville property if $u \in C_{l o c}^{1,1}\left(\mathbf{R}^{n}\right)$ is an entire viscosity solution of (1) with bounded $D^{2} u$ in $\mathbf{R}^{n},\left|D^{2} u\right| \leq C$, then $u$ must be a polynomial of degree at most 2 .

Let $B_{r}\left(x_{0}\right)=\left\{x \in \mathbf{R}^{n}:\left|x-x_{0}\right|<r\right\}$.

Now we state the main theorem.

Theorem. Suppose that $F \in C^{1}$ satisfies (2) and $F(0)=0$. Let $u \in C^{1,1}\left(B_{1}(0)\right)$ be a viscosity solution of (1) in $B_{1}(0)$. If equation (1) satisfies the Liouville property, then for any $0<\alpha<1, u \in C^{2, \alpha}\left(B_{1 / 2}(0)\right)$ and $\left[D^{2} u\right]_{C^{\alpha}\left(B_{1 / 2}(0)\right)} \leq C$, where $C$ depends only on $n, \lambda, \Lambda, \alpha,\|u\|_{C^{1,1}\left(B_{1}(0)\right)}, F$, and the modulus of continuity of $D F$.

\section{$\S 2$. The Proof of the Theorem}

We will use the blow-up technique to prove the Theorem. The tool needed to obtain a subsequence of blow-up solutions converging in $W_{l o c}^{2,2}\left(\mathbf{R}^{n}\right)$ is the $W^{2, \delta}$ estimate for nondivergent uniform elliptic equations. For the convenience of our readers, let us give a little more preliminary information.

Recall that $u \in \operatorname{BMO}(\Omega)$ is in $\operatorname{VMO}(\Omega)$ if

$$
\eta_{u}(R, \Omega)=\sup _{\substack{x_{0} \in \Omega \\ 0<r \leq R}} f_{B_{r}\left(x_{0}\right) \cap \Omega}\left|u(x)-u_{x_{0}, r}\right| d x \longrightarrow 0, \quad \text { as } R \longrightarrow 0,
$$

where $f_{A} f d x$ denotes the average of $f$ over $A$ and $u_{x_{0}, r}$ the average of $u$ over $B_{r}\left(x_{0}\right) \cap \Omega$. We will call $\eta_{u}$ the VMO modulus of $u$ in $\Omega$.

Now let us recall the class $\mathcal{S}$ of solutions of uniformly elliptic equations. For more details, see CC. Let $\mathcal{A}_{\lambda, \Lambda}$ denote all symmetric matrices whose eigenvalues belong to $[\lambda, \Lambda]$. Define Pucci extremal operators $M^{+}(M)$ and $M^{-}(M)$ by

$$
\begin{aligned}
& M^{+}(M)=\sup _{A \in \mathcal{A}_{\lambda, \Lambda}} \operatorname{trace}(A M), \\
& M^{-}(M)=\inf _{A \in \mathcal{A}_{\lambda, \Lambda}} \operatorname{trace}(A M),
\end{aligned}
$$

for $M \in \mathcal{S}$. It is easy to check that $M^{+}$and $M^{-}$are uniformly elliptic operators. A continuous function $u$ is in class $\mathcal{S}$ if $M^{-}\left(D^{2} u\right) \leq 0$ and $M^{+}\left(D^{2} u\right) \geq 0$ in the viscosity sense.

The following result on precompact sets in $L^{p}$ is a local variant of Theorem 3.44 in $\mathrm{A}]$.

Proposition 1. Let $\Omega$ be a bounded domain in $\mathbf{R}^{n}$ and $\mathcal{A}$ a bounded subset of $L^{p}(\Omega), 1 \leq p<\infty$. For any domain $D \subset \subset \Omega$, if

$$
\sup _{u \in \mathcal{A}} \int_{D}|u(x+h)-u(x)|^{p} d x \rightarrow 0, \quad \text { as }|h| \rightarrow 0,
$$

then $\mathcal{A}$ is precompact in $L^{p}(D)$.

Now let us prove the following lemma. 
Lemma 1. Assume that $F$ satisfies (2) and $F(0)=0$. Then the following two statements are equivalent:

(i) If $u \in C^{1,1}\left(B_{1}(0)\right)$ is a viscosity solution of (1) in $B_{1}(0)$ and $\left|D^{2} u\right| \leq M$ in $B_{1}(0)$, then $D^{2} u \in V M O\left(B_{1 / 2}(0)\right)$ and $\eta_{D^{2} u}(R) \leq \eta(R)$, where $\eta_{D^{2} u}(R)$ is the VMO modulus of $D^{2} u$ in $B_{1 / 2}(0), \lim _{R \rightarrow 0^{+}} \eta(R)=0$, and $\eta$ depends only on $n, \lambda, \Lambda, F$, and $M$.

(ii) F satisfies the Liouville property.

Proof. (i) implies (ii). Let $u \in C_{l o c}^{1,1}\left(\mathbf{R}^{n}\right)$ be an entire solution of (1) with $\left|D^{2} u\right| \leq M$ in $\mathbf{R}^{n}$. Consider

$$
v_{k}(y)=\frac{u(k y)-u(0)-D u(0) k y}{k^{2}}, \quad k=1,2, \cdots .
$$

Obviously $\left\|v_{k}\right\|_{C^{1,1}\left(B_{1}(0)\right)} \leq C_{n} M$ and

$$
F\left(D^{2} v_{k}\right)=0 \quad \text { in } B_{1}(0) .
$$

Therefore by (i), for $\rho>0$ we have

$$
\begin{aligned}
& f_{B_{\rho}(0)}\left|D^{2} u-\left(D^{2} u\right)_{0, \rho}\right| d x \\
= & f_{B_{\frac{\rho}{k}}(0)}\left|D^{2} v_{k}-\left(D^{2} v_{k}\right)_{0, \frac{\rho}{k}}\right| d y \\
\leq & \eta_{D^{2} v_{k}}\left(\frac{\rho}{k}\right) \leq \eta\left(\frac{\rho}{k}\right) \longrightarrow 0, \quad \text { as } k \longrightarrow \infty .
\end{aligned}
$$

This implies that $D^{2} u=$ const in $\mathbf{R}^{n}$ and hence $u$ is a polynomial of degree at most 2 .

Suppose that $F$ satisfies the Liouville property. We want to show (i). Let

$$
X_{M}=\left\{u \in C^{1,1}\left(B_{1}(0)\right): F\left(D^{2} u\right)=0 \text { and }\left|D^{2} u\right| \leq M \text { in } B_{1}(0)\right\} .
$$

To prove that (i) holds, it suffices to show the following claim:

$$
\sup _{\substack{u \in X_{M} \\ x_{0} \in B_{1 / 2}(0) \\ r \leq R}} f_{B_{r}\left(x_{0}\right)}\left|D^{2} u-\left(D^{2} u\right)_{x_{0}, r}\right|^{2} d x \longrightarrow 0, \quad \text { as } R \longrightarrow 0 .
$$

We will show (3) by contradiction. If (3) is false, then there exist $\varepsilon_{0}>0, r_{k} \longrightarrow 0$, $x_{k} \in B_{1 / 2}(0), u_{k} \in X_{M}$ such that for $k \geq 1$

$$
f_{B_{r_{k}}\left(x_{k}\right)}\left|D^{2} u_{k}-\left(D^{2} u_{k}\right)_{x_{k}, r_{k}}\right|^{2} d x \geq \varepsilon_{0} .
$$

Let

$$
\begin{gathered}
T_{k} y=x_{k}+r_{k} y, \quad \Omega_{k}=T_{k}^{-1} B_{1}(0) \\
v_{k}(y)=\frac{u_{k}\left(x_{k}+r_{k} y\right)-u_{k}\left(x_{k}\right)-D u_{k}\left(x_{k}\right) r_{k} y}{r_{k}^{2}} .
\end{gathered}
$$

It is easy to check that

$$
\begin{aligned}
& F\left(D^{2} v_{k}\right)=0, \quad \text { in } \Omega_{k} . \\
& f_{B_{1}(0)}\left|D^{2} v_{k}-\left(D^{2} v_{k}\right)_{0,1}\right|^{2} d y \geq \varepsilon_{0} . \\
& \left\|v_{k}\right\|_{C^{1,1}\left(B_{2 A}(0)\right)} \leq C_{n, A} M, \quad \text { if } B_{2 A r_{k}}\left(x_{k}\right) \subset B_{1}(0) .
\end{aligned}
$$


Now we want to show $\left\{D^{2} v_{k}\right\}$ is precompact in $L^{2}$. By [CC] (see Prop. 5.5)

$$
\triangle_{\tau e} v_{k}(y)=\frac{v_{k}(y+\tau e)-v_{k}(y)}{\tau} \in \mathcal{S}, \quad \text { in } B_{3 A / 2}(0),|e|=1 .
$$

By the $W^{2, \delta}$ estimate $(\delta>0)$ (see Prop. 7.4, $\mathrm{CC}$ ) for functions in $\mathcal{S}$

$$
\int_{B_{A}(0)}\left|D^{2} \triangle_{\tau e} v_{k}\right|^{\delta}(y) d y \leq C_{A}\left\|\triangle_{\tau e} v_{k}\right\|_{L^{\infty}\left(B_{3 A / 2}(0)\right)}^{\delta} \leq C_{A, M} M^{\delta},
$$

where $\delta$ and $C_{A}$ are independent of $k$.

Therefore, by (5) we have

$$
\begin{aligned}
& \int_{B_{A}(0)}\left|D^{2} v_{k}(y+\tau e)-D^{2} v_{k}(y)\right|^{2} d y \\
\leq & C\left\|D^{2} v_{k}\right\|_{L^{\infty}\left(B_{2 A}(0)\right)}^{2-\delta} \int_{B_{A}(0)}\left|D^{2} v_{k}(y+\tau e)-D^{2} v_{k}(y)\right|^{\delta} d y \\
\leq & C \tau^{\delta} .
\end{aligned}
$$

By Proposition 1, this fact together with

$$
\left\|D^{2} v_{k}\right\|_{L^{2}\left(B_{2 A}(0)\right)} \leq C_{A}
$$

implies that $\left\{D^{2} v_{k}\right\}$ is precompact in $L^{2}\left(B_{A}(0)\right)$. Since $v_{k}(0)=0, D v_{k}(0)=0$, and $\left\|v_{k}\right\|_{C^{1,1}\left(B_{2 A}(0)\right)} \leq C_{A}$, we may assume that by the diagonalizing process

$$
\begin{aligned}
& v_{k} \longrightarrow v, \quad \text { in } W_{l o c}^{2,2}\left(\mathbf{R}^{n}\right) \cap C_{l o c}^{1}\left(\mathbf{R}^{n}\right), \\
& D^{2} v_{k} \longrightarrow D^{2} v, \quad \text { a.e. in } \mathbf{R}^{n} .
\end{aligned}
$$

Therefore, $F\left(D^{2} v\right)=0$ in $\mathbf{R}^{n}$. Since $\left\|D^{2} v_{k}\right\|_{L^{\infty}\left(B_{A}(0)\right)} \leq\left\|D^{2} u_{k}\right\|_{L^{\infty}\left(B_{1}(0)\right)} \leq M$, $\left|D^{2} v\right| \leq M$ in $\mathbf{R}^{n}$. By the Liouville property, $v$ must be a polynomial of degree at most 2, and hence $D^{2} v=$ const. This contradicts the following:

$$
f_{B_{1}(0)}\left|D^{2} v-\left(D^{2} v\right)_{0,1}\right|^{2} d y=\lim _{k \rightarrow \infty} f_{B_{1}(0)}\left|D^{2} v_{k}-\left(D^{2} v_{k}\right)_{0,1}\right|^{2} d y \geq \varepsilon_{0} .
$$

Thus, Lemma 1 follows.

Lemma 2. Let $F \in C^{1}$ satisfy (2) and $F(0)=0$. If $u$ is a viscosity solution of (1) in $B_{1}(0)$ and $D^{2} u \in \operatorname{VMO}\left(B_{1}(0)\right)$, then for any $0<\alpha<1, u \in C^{2, \alpha}\left(B_{1 / 2}(0)\right)$ and $\left[D^{2} u\right]_{C^{2, \alpha}\left(B_{1 / 2}(0)\right)} \leq C$, where $C$ depends on $n, \alpha, \lambda, \Lambda$, the modulus of continuity of $D F,\|u\|_{C^{1}\left(B_{1}(0)\right)}$ and the VMO modulus of $D^{2} u$.

Proof. By differentiating (1), we obtain

$$
a_{i j}(x) D_{i j} \triangle_{h e} u(x)=0, \quad \text { in } B_{3 / 4}(0),
$$

where $\triangle_{h e} u(x)=[u(x+h e)-u(x)] / h, h<\frac{1}{4},|e|=1$, and

$$
a_{i j}(x)=\int_{0}^{1} \frac{\partial F}{\partial M_{i j}}\left((1-\theta) D^{2} u(x)+\theta D^{2} u(x+h e)\right) d \theta .
$$

Let $u_{h}(x)=u(x+h e)$ and

$$
c_{i j}=\int_{0}^{1} \frac{\partial F}{\partial M_{i j}}\left((1-\theta)\left(D^{2} u\right)_{x_{0}, r}+\theta\left(D^{2} u_{h}\right)_{x_{0}, r}\right) d \theta .
$$


Without loss of generality, we can assume that the continuity modulus of $D F$ denoted by $\omega(R)$ is concave. Obviously by Jensen's inequality

$$
\begin{aligned}
& f_{B_{r}\left(x_{0}\right)}\left|a_{i j}(x)-c_{i j}\right| d x \\
\leq & f_{B_{r}\left(x_{0}\right)} \int_{0}^{1} \omega\left[\left|(1-\theta)\left(D^{2} u-\left(D^{2} u\right)_{x_{0}, r}\right)+\theta\left(D^{2} u_{h}-\left(D^{2} u_{h}\right)_{x_{0}, r}\right)\right|\right] d \theta d x \\
\leq & \omega\left(\eta_{D^{2} u}(r)\right) \longrightarrow 0, \quad \text { as } r \longrightarrow 0 .
\end{aligned}
$$

Therefore $a_{i j} \in \operatorname{VMO}\left(B_{3 / 4}(0)\right)$. Since (2) holds, $\lambda^{\prime} I \leq\left(a_{i j}\right) \leq \Lambda^{\prime} I$ and (6) is uniformly elliptic. By the $L^{p}$ estimate in [CFL, we obtain

$$
\left\|\triangle_{h e} u\right\|_{W^{2, p}\left(B_{1 / 2}(0)\right)} \leq C\left\|\triangle_{h e} u\right\|_{L^{\infty}\left(B_{3 / 4}(0)\right)} .
$$

Thus, we finish the proof of Lemma 2 .

The Theorem follows immediately from Lemma 1 and Lemma 2.

\section{ACKNOWLEDGEMENT}

The author expresses his gratitude to Professor L. Caffarelli for discussions on several occasions. The author also thanks the referee for some suggestions.

\section{REFERENCES}

[A] T. Aubin, Nonlinear Analysis on Manifolds. Monge-Ampère Equations, Springer-Verlag, 1982. MR 85j:58002

[CC] L. Caffarelli \& X. Cabré, Fully nonlinear elliptic equations, AMS Colloquium Publications V. 43, AMS, Rhode Island, 1993. MR 96h:35046

[CFL] F. Chiarenza, M. Frasca \& P. Longo, $W^{2, p}$-solvability of the Dirichlet Problem for Nondivergence Elliptic Equations with VMO Coefficients, Trans. Amer. Math. Soc. 336 (1993), 841-853. MR 93f:35232

[GT] D. Gilbarg \& N. S. Trudinger, Elliptic partial differential equations of second order, 2nd edition, Springer-Verlag, 1983. MR 86c:35035

[Gi] M. Giaquinta \& J. Nečas, On the Regularity of Weak Solutions to Nonlinear Ellptic Systems of Partial Differential Equations, J. Reine Angew. Math. 316 (1980), 140-159. MR 81m:35056

[H] Q. Huang, Estimates on the Generalized Morrey Spaces $L_{\phi}^{2, \lambda}$ and $B M O_{\psi}$ for Linear Elliptic Systems, Indiana Univ. Math. J. 45 (1996), 397-439. MR 97i:35033

[K] N. V. Krylov, Nonlinear Elliptic and Parabolic Equations of Second Order, Mathematics and its Applications, Reidel, 1987. MR 88d:35005

[N] N. Nadirashvili, Nonclassical Solutions to Fully Nonlinear Elliptic Equations, Preprint.

Department of Mathematics, University of Texas at Austin, Austin, Texas 78712

E-mail address: qhuang@math.utexas.edu

Current address: Department of Mathematics \& Statistics, Wright State University, Dayton, Ohio 45435

E-mail address: qhuang@math.wright.edu 